\title{
Solution of velocity-vorticity URANS by BEM
}

\author{
L. Škerget \& J. Ravnik \\ Faculty of Mechanical Engineering, University of Maribor, Slovenia
}

\begin{abstract}
This paper describes the fundamental aspects of turbulence modelling for incompressible fluid flow and corresponding numerical models based on the boundarydomain integral equations. The velocity-vorticity formulation of the Reynolds averaged Navier-Stokes (RANS) equations is used, while the averaged pressure field is determined by solving the Poisson velocity equation. Several lowReynolds-number turbulent models, e.g. Launder-Sharma, are applied.
\end{abstract}

\section{Introduction}

The set of partial differential equations governing the motion of viscous fluid is known as nonlinear Navier-Stokes equations. This equation system is generally considered to be the fundamental description for all laminar as well as turbulent flows, although some statistical averaging procedure is required in practice to predict the turbulence and simulate numerically the flow at higher Reynolds number values due to the enormous computational effort needed. Turbulence is a highly complex transport phenomenon. A number of well established characteristics of a turbulent motion can be summarized as: highly nonlinear transport process, highly diffusive flow, three-dimensional flow, flow with multiple length and time scales, and time dependent (stochastic) transport phenomenon with identifiable statistical flow properties. In most cases of modelling and simulation of real engineering turbulent flows we are not interested in detailed resolution of all turbulent flow scales in favour of some type of averaged flow description. Therefore, turbulence effects enter the flow description via a model that is typically based on a combination of theory and experiment.

Various approaches exist for the turbulent flow prediction, i.e., full turbulence simulation, large eddy simulation, Reynolds averaged models, etc [1]. However, the extremely small time and length scales associated with turbulent fluid motion 
require impractically dense computational meshes and time steps when attempting to solve the unaveraged unsteady governing equations for practical engineering problems at realistic Reynolds number values.

The averaged form of the Navier-Stokes equations through the Reynolds decomposition of instantaneous value of each flow variable into a time averaged mean value, and an instantaneous deviation or fluctuation from the mean value is still the most commonly used approach in the numerical simulation of the engineering fluid flow problems with turbulence. The Reynolds mean flow equations involve more unknowns than equations. The term turbulence model implies assumptions, closing the mathematical description of the problem, i.e. makes the number of unknowns equal to the number of equations and enables the simulation of the flow in its important aspects. However, the computation of the turbulent flow based on any given turbulent model generally requires a knowledge of empirical constants.

\section{Governing equations}

\subsection{Primitive variables formulation}

When considering the turbulent flow a time averaged form of the Navier-Stokes equations is usually employed through the Reynolds decomposition of instantaneous value of each flow variable, e.g. velocity vector $v_{i}\left(r_{j}, t\right)$ into a time-averaged mean value $\overline{v_{i}}$ and an instantaneous deviation or fluctuation $v_{i}^{\prime}$ from the mean value, such that $v_{i}=\overline{v_{i}}+v_{i}^{\prime}$, and similarly pressure $p\left(r_{j}, t\right)$ into a time-averaged mean value $\bar{p}$ and an instantaneous deviation or fluctuation $p^{\prime}$ from the mean value, such that $p=\bar{p}+p^{\prime}$.

With the assumption of incompressibility the conservation equations set for the time-averaged mean field functions in a turbulent incompressible fluid motion, can be written as

$$
\begin{gathered}
\frac{\partial \overline{v_{j}}}{\partial x_{j}}=0 \\
\rho_{o} \frac{D \bar{v}_{i}}{D t}=-\frac{\partial \bar{p}}{\partial x_{i}}-\frac{\partial \overline{\tau_{i j}}}{\partial x_{j}}+\rho_{o} g_{i}
\end{gathered}
$$

for $i, j=1,2,3$.

The time-averaged mean momentum flux tensor $\overline{\tau_{i j}}$ can be written as a sum of molecular diffusion flux for the time mean values and the turbulent flux, e.g. in the form

$$
\overline{\tau_{i j}}={\overline{\tau_{i j}}}^{m}+{\overline{\tau_{i j}}}^{t}=-2 \eta_{o}{\overline{\dot{\varepsilon}_{i j}}}+\rho_{o} \overline{v_{i}^{\prime} v_{j}^{\prime}},
$$

in which the tensor quantity $\overline{\dot{\varepsilon}_{i j}}$ is the symmetrical part of the time-averaged mean velocity gradient

$$
\overline{\dot{\varepsilon}_{i j}}=\frac{1}{2}\left(\frac{\partial \overline{v_{i}}}{\partial x_{j}}+\frac{\partial \overline{v_{j}}}{\partial x_{i}}\right) .
$$

The Reynolds averaged Navier-Stokes (RANS) equations set, governing the transport of time mean flow quantities in a turbulent incompressible fluid flow, 
can be obtained substituting eq.(3) in eq.(2), as follows

$$
\begin{gathered}
\frac{\partial \overline{v_{j}}}{\partial x_{j}}=0 \\
\rho_{o} \frac{D \overline{v_{i}}}{D t}=-\frac{\partial \bar{p}}{\partial x_{i}}+\frac{\partial}{\partial x_{j}}\left(2 \eta_{o} \overline{\dot{\varepsilon}_{i j}}\right)+\rho_{o} g_{i}-\frac{\partial \rho_{o} \overline{v_{i}^{\prime} v_{j}^{\prime}}}{\partial x_{j}},
\end{gathered}
$$

With the exception of the additional Reynolds stress tensor or turbulent momentum flux tensor $\tau_{i j}^{t}$, the turbulent heat flux vector $q_{i}^{t}$, and the turbulent mass flux vector $j_{i}^{t}$

$$
\tau_{i j}^{t}=\rho_{o} \overline{v_{i}^{\prime} v_{j}^{\prime}}=\rho_{o} \overline{\vec{v}^{\prime} \otimes \vec{v}^{\prime}}
$$

the mean flow quantities of a turbulent flow and the instantaneous flow quantities of a laminar flow satisfy the same set of differential equations; the no-slip condition on the solid surfaces and the free-stream condition for external flows, satisfied by the instantaneous velocity of the laminar flow, are also satisfied by the mean velocity of a turbulent flow.

\subsection{Turbulence models}

The completion of the mean flow governing equations requires that additional constitutive relations for the Reynolds fluxes be provided. This specification constitutes a turbulence model. An enormous variety of turbulence models exist, ranging in complexity from simple algebraic relations to descriptions involving multiple, nonlinear governing partial differential transport equations for specific turbulent quantities [2-4].

As noted previously, the term $\rho_{o} \overline{v_{i}^{\prime} v_{j}^{\prime}}$ appearing on the right hand side of eq.(6) is due to turbulent motion and is called the Reynolds stress. It acts to promote the diffusion of momentum and cannot be obtained by further time-averaging alone and has to be modelled. The main aim of all turbulence models is to find a rational closure of equations; any turbulent model requires a knowledge of empirical constants.

By analogy with the molecular momentum diffusion, the Boussinesq approximation expressed the turbulent momentum transport in terms of mean velocity gradients

$$
\tau_{i j}^{t}=\rho_{o} \overline{v_{i}^{\prime} v_{j}^{\prime}}=-2 \eta_{t} \overline{\dot{\varepsilon}_{i j}}+\frac{2}{3} \rho_{o} k \delta_{i j}=-\rho_{o} \nu_{t}\left(\frac{\partial \bar{v}_{i}}{\partial x_{j}}+\frac{\partial \bar{v}_{j}}{\partial x_{i}}\right)+\frac{2}{3} \rho_{o} k \delta_{i j},
$$

where $\nu_{t}=\eta_{t} / \rho_{o}$ is the eddy-viscosity or turbulent viscosity computed by the chosen turbulence model, and $k$ mean turbulent kinetic energy. Unlike the molecular momentum diffusivity coefficient $\nu$ which is a fluid property, the turbulent (eddy) diffusivity coefficient $\nu_{t}$ is local property of turbulent flow. Models for the turbulent transport properties attempt to produce reasonable turbulent effects within the governing equations as functions of the mean flow quantities. 


\subsection{Reynolds averaged Navier-Stokes mean flow equations}

When the definitions in eq.(8) are substituted into governing eq.(6) the equations for the mean flow can be written in terms of effective or total momentum diffusivity $\nu_{e f}=\nu+\nu_{t}$, as follows

$$
\begin{gathered}
\frac{\partial \overline{v_{j}}}{\partial x_{j}}=0 \\
\rho_{o} \frac{D \overline{v_{i}}}{D t}=-\frac{\partial \bar{p}^{\star}}{\partial x_{i}}+\frac{\partial}{\partial x_{j}}\left(\rho_{o} 2 \nu_{e f} \overline{\dot{\varepsilon}_{i j}}\right)+\rho_{o} g_{i},
\end{gathered}
$$

where the volumetric part of Reynolds stress has been included in the pressure term such as

$$
\bar{p}^{\star}=\bar{p}+\frac{2}{3} \rho_{o} k .
$$

The momentum equation eq.(10) can be written in a extended form suitable for velocity-vorticity formulation, e.g. in Cartesian tensor notation formulation

$$
\rho_{o} \frac{D \overline{v_{i}}}{D t}=-e_{i j k} \frac{\partial \overline{\omega_{k}}}{\partial x_{j}} \eta_{e f}+e_{i j k} \frac{\partial \eta_{e f}}{\partial x_{j}} \overline{\omega_{k}}+2 \frac{\partial \eta_{e f}}{\partial x_{j}} \frac{\partial \overline{v_{i}}}{\partial x_{j}}-\frac{\partial \bar{p}^{\star}}{\partial x_{i}}+\rho_{o} g_{i} .
$$

Representing the effective transport properties, e.g. the effective dynamic viscosity $\eta_{e f}$ as a sum of a constant and variable part

$$
\eta_{e f}=\eta_{e f o}+\widetilde{\eta_{e f}}
$$

then the momentum eq.(12) can be written in analogy to the basic conservation equations formulated for the constant material properties

$$
\rho_{o} \frac{D \overline{\vec{v}}}{D t}=-\eta_{e f o} \operatorname{rot} \overline{\vec{\omega}}-\operatorname{grad} \bar{p}^{\star}+\rho_{o} \vec{g}+\overline{\vec{f} m},
$$

where the pseudo body force term $\overline{\vec{f}^{m}}$ is introduced into the momentum eq.(14) capturing the variable transport property effects, and given by expression

$$
\overline{f_{i}^{m}}=-e_{i j k} \frac{\partial \overline{\omega_{k}}}{\partial x_{j}} \widetilde{\eta_{e f}}+e_{i j k} \frac{\partial \eta_{e f}}{\partial x_{j}} \overline{\omega_{k}}+2 \frac{\partial \eta_{e f}}{\partial x_{j}} \frac{\partial \overline{v_{i}}}{\partial x_{j}}
$$

which for plane flow problems reduces to relation

$$
\overline{f_{i}^{m}}=-e_{i j} \frac{\partial \bar{\omega}}{\partial x_{j}} \widetilde{\eta_{e f}}+e_{i j} \frac{\partial \eta_{e f}}{\partial x_{j}} \bar{\omega}+2 \frac{\partial \eta_{e f}}{\partial x_{j}} \frac{\partial \overline{v_{i}}}{\partial x_{j}} .
$$

Once the form of the eddy diffusivity coefficients are specified then the mean transport equations can be solved in the same manner as a laminar flow since the equations are the same except for augmented diffusivity coefficients. Though the turbulent flow problem has been reduced to a familiar system of partial differential transport equations, there remains the nontrivial task of determining how the eddy diffusivity coefficients vary with the flow field. 


\subsection{Velocity-vorticity mean flow formulation}

With the mean vorticity vector $\overline{\omega_{i}}$ representing the curl of the mean velocity field $\overline{v_{i}}$

$$
\overline{\omega_{i}}=e_{i j k} \frac{\partial \overline{v_{k}}}{\partial x_{j}} \quad \text { and } \quad \frac{\partial \overline{\omega_{j}}}{\partial x_{j}}=0,
$$

the fluid motion computation scheme is partitioned into its kinematics, given by the elliptic mean velocity vector equation

$$
\frac{\partial^{2} \overline{v_{i}}}{\partial x_{j} \partial x_{j}}+e_{i j k} \frac{\partial \overline{\omega_{k}}}{\partial x_{j}}=0,
$$

and kinetics given by mean vorticity transport equation, obtained as a curl of the mean momentum eq.(16), e.g., written in Cartesian tensor notation formulation

$$
\frac{\partial \overline{\omega_{i}}}{\partial t}+\frac{\partial \overline{v_{j}} \overline{\omega_{i}}}{\partial x_{j}}=\nu_{e f o} \frac{\partial^{2} \overline{\omega_{i}}}{\partial x_{j} \partial x_{j}}+\frac{\partial \overline{\omega_{j}} \overline{v_{i}}}{\partial x_{j}}+\frac{1}{\rho_{o}} e_{i j k} \frac{\partial \overline{f_{k}^{m}}}{\partial x_{j}},
$$

which reduces for two-dimensional plane flow case, to the following scalar mean vorticity statement

$$
\frac{\partial \bar{\omega}}{\partial t}+\frac{\partial \overline{v_{j}} \bar{\omega}}{\partial x_{j}}=\nu_{e f o} \frac{\partial^{2} \bar{\omega}}{\partial x_{j} \partial x_{j}}-\frac{1}{\rho_{o}} e_{i j} \frac{\partial \overline{f_{i}^{m}}}{\partial x_{j}} .
$$

The vorticity transport eq.(19) is highly nonlinear partial differential equation. Due to the buoyancy force and variable effective transport properties, acting as additional nonlinear vorticity source terms, the vorticity transport equation is coupled to the energy/mass and transport equations for the turbulence quantities, i.e., ' $k$ ' and ' $e$ ' transport equations, making the numerical computation procedure very challenging.

\section{Eddy-viscosity turbulence models}

\subsection{Two-equation LRN $k-\epsilon$ turbulence models}

Low-Reynolds-number form turbulence models [5-7] seem to be necessary for accurate prediction of unsteady turbulent wall boundary flows where the near-wall physics is of interest. In an unsteady turbulent wall bounded flow, the near-wall region is characterized by a rapid phase change in flow quantities. The phase shift in the wall shear stress is important in engineering applications. Since at high frequencies the thickness of the unsteady layer can be of the same order of the viscous sublayer, incorrect near-wall asymptotic behaviour of a turbulence model may result in inaccurate prediction of near-wall unsteady turbulent stresses. In the vicinity of the wall, the presence of the solid wall causes preferential damping of the normal component of turbulent velocity. At the same time, the turbulence motion is restricted and the Reynolds number becomes small. 
In the $k-\epsilon$ turbulence models, the turbulent motion is characterised by two quantities, namely the turbulent kinetic energy $k$ and the turbulent energy dissipation rate $\epsilon$, which extracts energy from the turbulence and converts this energy to heat. These values are given by relations

$$
\begin{gathered}
k=\frac{1}{2} \overline{v_{i}^{\prime} v_{i}^{\prime}}, \\
\epsilon=\nu \overline{\frac{\partial v_{i}^{\prime}}{\partial x_{j}} \frac{\partial v_{i}^{\prime}}{\partial x_{j}}},
\end{gathered}
$$

while the turbulent viscosity is given by the Kolmogorov-Prandtl relation

$$
\eta_{t}=C_{\eta} f_{\eta} \frac{k^{2}}{\epsilon},
$$

which relates the eddy viscosity directly to the turbulence variables, $k$ and $\epsilon$, and where $C_{\eta}=0.09$ is an empirical constant. The function $f_{\eta}$ is needed to interface the near-wall low-Reynolds-number eddy viscosity on the high-Reynolds-number eddy viscosity.

Both turbulent quantities $k$ and $\epsilon$ are determined from individual transport equations. The equations have also to be modelled, which means that several assumptions have to be considered in order to close the mathematical description, since these equations involve moments of fluctuating velocity of order higher than two. Since the turbulent fluctuations are damped near a solid wall, there is a region close to the wall where viscous effects are important. In this region, the local turbulence Reynolds number values, defined by the expressions

$$
\begin{array}{r}
R e_{t}=\frac{\rho k^{2}}{\eta \epsilon}, \quad R e_{k}=\frac{\rho \sqrt{k} y}{\eta}, \\
R e_{\tau}=\frac{\rho v_{\tau} y}{\eta}=y^{+}, \quad R e_{K}=\frac{\rho v_{K} y}{\eta}=y^{*},
\end{array}
$$

where $\eta$ is the molecular dynamic viscosity of the fluid, $y^{+}$and $y^{*}$ are the dimensionless normal distances from wall based on the wall friction velocity $v_{\tau}=$ $\sqrt{\tau_{w} / \rho}=\sqrt{\nu|\omega|}$ and Kolmogorov velocity scale $v_{K}=(\nu \epsilon)^{1 / 4}$, respectively, are small and the high Reynolds number turbulence models are no longer applicable. For this reason, low-Reynolds-number (LRN) $k-\epsilon$ turbulence models can be employed allowing integration right down to the wall with appropriate boundary conditions. This requires more grid nodes in the near-wall region than the other two schemes described above, but does permit a continuous solution without the problem of specifying an artificial match node. The damping of turbulence near a solid wall due to molecular diffusion is simulated through some damping functions attached to various terms of transport equations for the specific turbulent quantities in the region which allow a smooth change of the flow variable from the small viscous sublayer values very near the wall to the fully turbulent value away from the wall. 
In the two equation $k-\epsilon$ low-Reynolds-number models with linear eddyviscosity models, the values of $k$ and $\epsilon$ come directly from the differential transport equations for the turbulent kinetic energy and the eddy dissipation rate

$$
\begin{gathered}
\rho_{o} \frac{D k}{D t}=\frac{\partial}{\partial x_{j}}\left[\left(\eta_{o}+\frac{\eta_{t}}{\sigma_{k}}\right) \frac{\partial k}{\partial x_{j}}\right]+P_{k}-\rho_{o} \widetilde{\epsilon}-\rho_{o} D, \\
\rho_{o} \frac{D \widetilde{\epsilon}}{D t}=\frac{\partial}{\partial x_{j}}\left[\left(\eta_{o}+\frac{\eta_{t}}{\sigma_{\epsilon}}\right) \frac{\partial \widetilde{\epsilon}}{\partial x_{j}}\right]+C_{\epsilon 1} f_{\epsilon 1} \frac{\widetilde{\epsilon}}{k} P_{k}-C_{\epsilon 2} f_{\epsilon 2} \rho_{o} \frac{\widetilde{\epsilon}^{2}}{k}+\rho_{o} E,
\end{gathered}
$$

where the new model variable $\widetilde{\epsilon}$ introduced by Launder and Sharma [8] is defined as

$$
\epsilon \equiv \widetilde{\epsilon}+D \quad \text { with } \quad D=2.0 \nu_{o}\left(\frac{\partial k^{1 / 2}}{\partial x_{j}}\right)^{2} .
$$

Since the dissipation rate at the wall is equal to

$$
\left.\left.\epsilon\right|_{\text {wall }} \equiv D\right|_{\text {wall }}=\left.2.0 \nu_{o}\left(\frac{\partial k^{1 / 2}}{\partial x_{j}}\right)^{2}\right|_{\text {wall }},
$$

the variable $\tilde{\epsilon}$ is zero at the wall which simplifies the specification of wall boundary conditions, i.e.

$$
k=\widetilde{\epsilon}=0 .
$$

Further, such definition of new variable $\tilde{\epsilon}$ also gives rise to an extra term $E$ in the $\widetilde{\epsilon}$ transport equation

$$
E=2.0 \nu_{o} \nu_{t}\left(\frac{\partial^{2} \overline{v_{i}}}{\partial x_{j} \partial x_{k}}\right)^{2} .
$$

The turbulent kinetic energy production term $P_{k}$ is due to viscous forces and is modeled, e.g. by the following relation

$$
P_{k}=2 \eta_{t} \overline{\dot{\varepsilon}_{i j}} \frac{\partial \overline{v_{i}}}{\partial x_{j}}=\eta_{t}\left(\frac{\partial \overline{v_{i}}}{\partial x_{j}}+\frac{\partial \overline{v_{j}}}{\partial x_{i}}\right) \frac{\partial \overline{v_{i}}}{\partial x_{j}} .
$$

One of the most popular and well tested LRN $k-\widetilde{\epsilon}$ is the Launder and Sharma model (LS), where the damping functions are expressed as functions of the local turbulence Reynolds number as follows:

$$
\begin{gathered}
f_{\eta}=\exp \left[-\frac{3.4}{\left(1+0.02 R e_{t}\right)^{2}}\right], \\
f_{\epsilon 1}=1 \quad \text { and } \quad f_{\epsilon 2}=1.00-0.3 \exp \left(-R e_{t}^{2}\right),
\end{gathered}
$$

with $C_{\epsilon 1}=1.44, C_{\epsilon 2}=1.92, \sigma_{k}=1.0$, and $\sigma_{\epsilon}=1.3$, while $\sigma_{\rho}=0.9$, respectively.

The two equation, $k-\epsilon$ turbulence model described by eqs.(25) and (26) can be used in conjunction with the mean flow equations and the definition of $\eta_{t}$ given by eq.(23), to arrive at a continuum description of turbulent motion. This system 
of equations is highly nonlinear, with a strong coupling among various transport equations. Although, the model is far from being universal and has a number of weaknesses, it remains one of the most frequently used for real turbulent flow computation. It is also evident, that the size of the computational problem and numerical difficulties for a two equation turbulent simulation increases substantially over its laminar counterpart.

\section{Numerical aspects: iterative strategy}

Once a turbulence model has been selected for a specific application, it is important to consider how a numerical model, e.g. boundary element method, would be applied to produce correct flow simulation. The boundary element implementation of the two equation $k-\epsilon$ low-Reynolds-number model is basically straightforward since the transport equations for the turbulent quantities are of the familiar diffusion-convection type. The boundary element solution of these types of equations and of the velocity-vorticity formulation of Navier-Stokes equations was presented in [9-12].

The discretised forms of eqs.(25) and (26) are highly nonlinear and strongly coupled to the mean flow RANS equations. It is also evident that the computational effort for a two equation turbulent simulation has increased substantially over its laminar counterpart. At the same time, the numerical difficulties have also increased significantly mainly due to two reasons: the nonlinear behaviour of the transport equations and boundary conditions for the turbulent transport and RANS partial differential equations. In particular, the dissipation equation may cause instabilities that lead to poor or nonconvergence of the numerical solution procedure. Stability problems can result in the prediction of negative values for both turbulent quantities $k$ and $\epsilon$. This non-physical behaviour of turbulent field functions can be also caused by a inadequate modelling of the source terms for $k$ and $\epsilon$. In many cases, the problem can be overcome by the clipping procedure in which negative values are replaced by small positive values.

Further, due to very sensitive nature of the $k$ and $\epsilon$ transport equations, it is of main importance to establish stable solution procedure. Generally, Picard or simple under-relaxation iteration technique should be applied in order to solve these coupled equations. The convergence of the $k$ and $\epsilon$ field functions is in general very slow and may not be monotonic. Though the convergence rate may be improved by the linearization of the $k$ and $\epsilon$ transport equations.

The solution iterative strategy is to solve for velocity $\vec{v}$ and vorticity $\vec{\omega}$ field functions and then to solve for $k$ and $\epsilon$ until convergence, repeating the iterative process if needed. The solution scheme is as follows:

1. Solve the Reynolds averaged Navier-Stokes equations

2. Solve for $k$ and $\epsilon$

2.1 Update production $P_{k}$ and $P_{\epsilon}$

2.2 Solve for $k$ - (until convergence) 


\subsection{Solve for $\epsilon$ - (until convergence)}

2.4 Check convergence in terms of $\nu_{t}$. If not, go to 2.2.

3. Check convergence for $\vec{\omega}$. If not, go to 1 .

For the given velocity field $\vec{v}$ the $k$ and $\epsilon$ equations have to be solved. The first point of the iterative scheme is that the equations for $k$ and $\epsilon$ are coupled iteratively. Therefore, the nonlinear transport equation is solved for $k$ first assuming that $\epsilon$ is known and then the computed value of $k$ is used to solve the nonlinear transport equation for $\epsilon$. Since it is not desired to deal with problems with a negative production term, the term $P_{k}$ is kept constant in eq.(25) and also production term $P_{\epsilon}$, i.e.

$$
P_{\epsilon}=C_{\epsilon 1} f_{\epsilon 1} \frac{\epsilon}{k} P_{k}=C_{\epsilon 1} f_{\epsilon 1} \frac{P_{k}}{\tau_{t}},
$$

is kept constant in eq.(26).

Let us first consider eq.(25). In order to avoid keeping $\epsilon$ constant, the dissipation term is written as

$$
D_{k}=\epsilon=\frac{C_{\eta} f_{\eta}}{\nu_{t}} k^{2} .
$$

Therefore, in the innermost iterative loop the linearized $k$ equation is solved, e.g. for given $\nu_{t}$ and $P_{k}$ solve until converge

$$
\rho_{o} \frac{D k_{i}}{D t}=\frac{\partial}{\partial x_{j}}\left[\left(\eta_{o}+\frac{\eta_{t}}{\sigma_{k}}\right) \frac{\partial k_{i}}{\partial x_{j}}\right]+P_{k}-\rho_{o} \frac{C_{\eta} f_{\eta}}{\nu_{t}}\left(2 k_{i-1} k_{i}-k_{i-1}^{2}\right),
$$

where the subscript stands for the iteration counter.

Similarly, the following linearized $\epsilon$ equation is solved, e.g. for given $\nu_{t} k$, and $P_{\epsilon}$ solve until converge

$$
\rho_{o} \frac{D \epsilon_{i}}{D t}=\frac{\partial}{\partial x_{j}}\left[\left(\eta_{o}+\frac{\eta_{t}}{\sigma_{\epsilon}}\right) \frac{\partial \epsilon_{i}}{\partial x_{j}}\right]+P_{\epsilon}-\rho_{o} \frac{C_{\epsilon 2} f_{\epsilon 2}}{k}\left(2 \epsilon_{i-1} \epsilon_{i}-\epsilon_{i-1} \epsilon_{i-1}\right) .
$$

It can be observed, that linearized $k$ and $\epsilon$ eqs.(36) and (37) have the same structure. Now, the iterative scheme can be written in detail, as follows:

1. Compute $P_{k}$ and $P_{\epsilon}$

2. Repeat until convergence for $\nu_{t}$

2.1 Solve for $k$ :

2.1.1 Solve eq.(25)

2.1.2 $k_{i}=u r \cdot k_{i}+(1-u r) \cdot k_{i-1}$

2.2 Check convergence for $k$. If not, go to 2.1.

2.3 Update $\nu_{t}$ using eq.(23)

2.4 Solve for $\epsilon$ :

2.4.1 Solve eq.(26)

2.4.2 $\epsilon_{i}=u r \cdot \epsilon_{i}+(1-u r) \cdot \epsilon_{i-1}$

2.4 Check convergence for $\epsilon$. If not, go to 2.4.

2.5 Update $\nu_{t}$ using eq.(23)

3. Check convergence for $\nu_{t}$. If not, go to 2 . 
In monitoring the convergence of a turbulent flow simulation it is noteworthy that the velocity and vorticity field functions converge first. The grading of the computational mesh may have also a big impact on the solution stability, e.g., abrupt changes in mesh density can lead to divergence of the $k$ and $\epsilon$ solution process.

The discretization in generally greatly affects the numerical results, and this is especially true for the low-Reynolds turbulent models. The near-wall region where large gradients of the field functions occur has to be adequately discretized; the first internal nodes have to be placed into the viscous sublayer, e.g., of the order $y^{+}=0.5$ from the solid wall.

\section{Boundary conditions for turbulent flows}

In order to solve the complete dynamical coupled nonlinear equations system, some physically justified boundary conditions must be specified. Especially troublesome in this regard are $k$ and $\epsilon$ values for inlet boundaries, since to a large extent these values will set the turbulence level for the entire flow field. In general, boundary conditions assigned to diffusion-convective transport equations are of the first, second, mixed and outflow-convective type, imposed on the part of the boundary.

The boundary conditions applied at the specific parts of the surface are as follows, e.g., specified Dirichlet boundary conditions for the variables $\left(\overline{v_{i}}, \bar{k}, \overline{\widetilde{\epsilon}}\right)$ at the inflow boundaries

$$
\overline{v_{i}}=\overline{\overline{v_{i}}}, \quad k=\bar{k} \quad \text { and } \quad \widetilde{\epsilon}=\overline{\widetilde{\epsilon}},
$$

zero Dirichlet values for the functions $\left(\overline{v_{i}}, \bar{k}, \overline{\widetilde{\epsilon}}\right)$ at the solid wall boundaries, no-slip conditions,

$$
\overline{v_{i}}=\bar{k}=\overline{\widetilde{\epsilon}}=0,
$$

or zero Dirichlet values for the functions $\left(\overline{v_{i}}, \bar{k}\right)$ and zero Neumann for $\bar{\epsilon}$ at the solid wall boundaries

$$
\overline{v_{i}}=\bar{k}=0 \quad \text { and } \quad \frac{\overline{\partial \epsilon}}{\partial n}=0
$$

and the outflow boundary conditions for the field quantities $(k, \widetilde{\epsilon})$ as zero Neumann normal flux values

$$
\frac{\partial k}{\partial n}=\frac{\partial \widetilde{\epsilon}}{\partial n}=0
$$

or in the form of outflow-convective boundary conditions

$$
\frac{\partial k}{\partial t}+\langle\bar{v}\rangle \frac{\partial k}{\partial n}=0 \quad \text { and } \quad \frac{\partial \widetilde{\epsilon}}{\partial t}+\langle\bar{v}\rangle \frac{\partial \widetilde{\epsilon}}{\partial n}=0
$$

where $\langle\bar{v}\rangle$ is the mean outflow velocity. 


\section{Conclusion}

In this work a numerical procedure based on the boundary element method for the simulation of two-dimensional is presented. Low Reynolds number $k-\epsilon$ has been considered. Iterative strategy of highly nonlinear and coupled governing equations is discussed. Future work will include two standard examples, e.g. fully developed turbulent flow [13-15] in channel and turbulent backward facing step flow [16], to test the proposed numerical algorithm. The nonlinearity of low Reynolds models is very severe and special attention will have to be paid to obtain convergence numerical solution.

\section{References}

[1] Biswas, G. \& Eswaran, V., Turbulent Flows: Fundamentals, Experiments and Modeling. Alpha Science International Ltd., Pangbourne, United Kingdom, 2002.

[2] Nagano, Y. \& Hishida, M., Improved Form of the $k-\epsilon$ Model for Wall Turbulent Shear Flows. ASME Journal of Fluids Engineering, 109, pp. 156160, 1987.

[3] Nagano, Y. \& Kim, C., A Two-Equation Model for Heat Transport in Wall Turbulent Shear Flows. ASME Journal of Heat Transfer, 110, pp. 583-589, 1988.

[4] Nagano, Y. \& Tagawa, M., An Improved $k-\epsilon$ Model for Boundary Layer Flows. ASME Journal of Fluids Engineering, 112, pp. 33-39, 1990.

[5] Patel, V., Rodi, W. \& Scheurer, G., Evaluation of Turbulence Models for Near-Wall and Low-Reynolds Number Flows. 3rd Symposium on Turbulent Shear Flows, University of California, 1981.

[6] Chien, K., Predictions of Channel and Bounday-Layer Flows with a LowReynolds-Number Turbulence Model. AIAA Journal, 20, pp. 33-38, 1982.

[7] Fan, S. \& Lakshminarayana, B., Low-Reynolds-Number $k-\epsilon$ Model for Unsteady Turbulent Boundary-Layer Flows. AIAA Journal, 31, pp. 17771784, 1993.

[8] Launder, J. \& Sharma, A first Course in Turbulence. MIT Press: Boston, 1974.

[9] Škerget, L. \& Ravnik, J., BEM simulation of compressible fluid flow in an enclosure induced by thermoacoustic waves. Eng Anal Bound Elem, 33, pp. 561-571, 2009.

[10] Škerget, L., Hriberšek, M. \& Žunič, Z., Natural convection flows in complex cavities by BEM. Int J Num Meth Heat \& Fluid Fl, 13, pp. 720-735, 2003.

[11] Popov, V., Power, H. \& Škerget, L., (eds.) Domain Decomposition Techniques for Boundary Elements: Applications to fluid flow. WIT press, 2007.

[12] Ravnik, J., Škerget, L. \& Hriberšek, M., 2D velocity vorticity based LES for the solution of natural convection in a differentially heated enclosure by wavelet transform based BEM and FEM. Eng Anal Bound Elem, 30, pp. 671686, 2006. 
40 Boundary Elements and Other Mesh Reduction Methods XXXII

[13] Moin, P. \& Kim, J., Numerical investigation of turbulent channel flow. J Fluid Mech, 118, pp. 341-377, 1982.

[14] Kim, J., Moin, P. \& Moser, R., Turbulence statistics in fully developed channel flow at low Reynolds number. J Fluid Mech, 177, pp. 133-166, 1987.

[15] Kim, S., Near-Wall Turbulence Model and its Application to Fully Developed Turbulent Channel and Pipe Flows. Numerical Heat Transfer,Part B, 17, pp. 101-122, 1990.

[16] Ramšak, M. \& Škerget, L., A multidomain boundary element method for two equation turbulence models. Eng Anal Bound Elem, 29, pp. 1086-1103, 2005. 